\title{
La importancia del liderazgo y su influencia en el personal para el cumplimiento de metas organizacionales
}

\section{The importance of leadership and its influence on staff for the fulfilment of organizational goals}

\author{
Ruth D. Ambrosio-Osorio ${ }^{a}$, Naomi Ascencio-Hernández ${ }^{b}$, Andrea G. Cano-Ramírez ${ }^{c}$, Daniela \\ J. Peralta-Santiago ${ }^{d}$, Daniela Ramírez-García ${ }^{e}$, Steven Valdez-Vázquez ${ }^{f}$
}

\begin{abstract}
:
When talking about organizational goals, we refer to those objectives that are interested to fulfill anybody (call it a company, organization or even business) dedicated to work with people helping fulfill them. It focuses on investigating; what type of leadership influences people to achieve objectives? In addition to what is important to the organization? Seen from a theoretical perspective.
\end{abstract}

\section{Keywords:}

Goals, influence, leadership, people

\section{Resumen:}

Al hablar de metas organizacionales nos referimos aquellos objetivos que le interesa cumplir a cualquier órgano (llámese empresa, organización incluso negocio) que se dedique a trabajar con personas que ayuden en el cumplimiento de las mismas, el presente trabajo se enfoca en indagar en las siguientes dos interrogantes ¿Cómo es que el tipo de liderazgo influye en las personas para alcanzar objetivos? además de ¿Cuál es su importancia dentro de la organización? visto desde una perspectiva teórica.

\section{Palabras Clave:}

Metas, influencia, liderazgo, personal

\section{Introducción}

Al definir el liderazgo, identificamos que se hablaba de una habilidad (que puede o no ser innata del ser humano) de influir en el actuar de otras personas, con el fin que estas hagan lo que el líder desea que realicen por y para el grupo. A la par que el liderazgo no es el mismo en todas las organizaciones ni en todas las situaciones, por ejemplo, el liderazgo empleado por un oficial de la marina no puede ser el mismo que un docente ejerce en el salón de clases; a pesar de ser una habilidad que en teoría suele tener siempre el mismo fin, en la práctica resulta complicado llevar de la misma manera por todos los líderes.

Entonces nos preguntamos ¿Cómo se sienten los liderados al respecto? ¿El tipo de liderazgo estará directamente relacionado con el cumplimiento de objetivos?, sin embargo, había una cosa más que abordar; ¿Por qué el liderazgo es importante realmente? ¿Acaso los grupos necesitan un líder que guíe sus acciones o simplemente lo necesitamos por mera costumbre?

En la siguiente infografía se tratará de identificar la forma en que algunos estilos de liderazgo influyen directa $o$ indirectamente en el cumplimiento de

\footnotetext{
${ }^{a}$ Universidad Autónoma del Estado de Hidalgo, https://orcid.org/0000-0002-7221-8258, Email: am441289@uaeh.edu.mx

b Universidad Autónoma del Estado de Hidalgo, https://orcid.org/0000-0002-9714-4945, Email: as440163@uaeh.edu.mx

c Universidad Autónoma del Estado de Hidalgo, https://orcid.org/0000-0002-7337-6098, Email: ca440146@uaeh.edu.mx

d Universidad Autónoma del Estado de Hidalgo, https://orcid.org/0000-0003-3327-3446, Email: pe440114@uaeh.edu.mx

e Universidad Autónoma del Estado de Hidalgo, https://orcid.org/0000-0003-3422-2050, Email: ra442991@uaeh.edu.mx
}

${ }^{\mathrm{f}}$ Autor de correspondencia, Universidad Autónoma del Estado de Hidalgo, https://orcid.org/0000-0002-4525-7657, Email: va379540@uaeh.edu.mx 
objetivos, así como la forma en que lo hace en las personas.

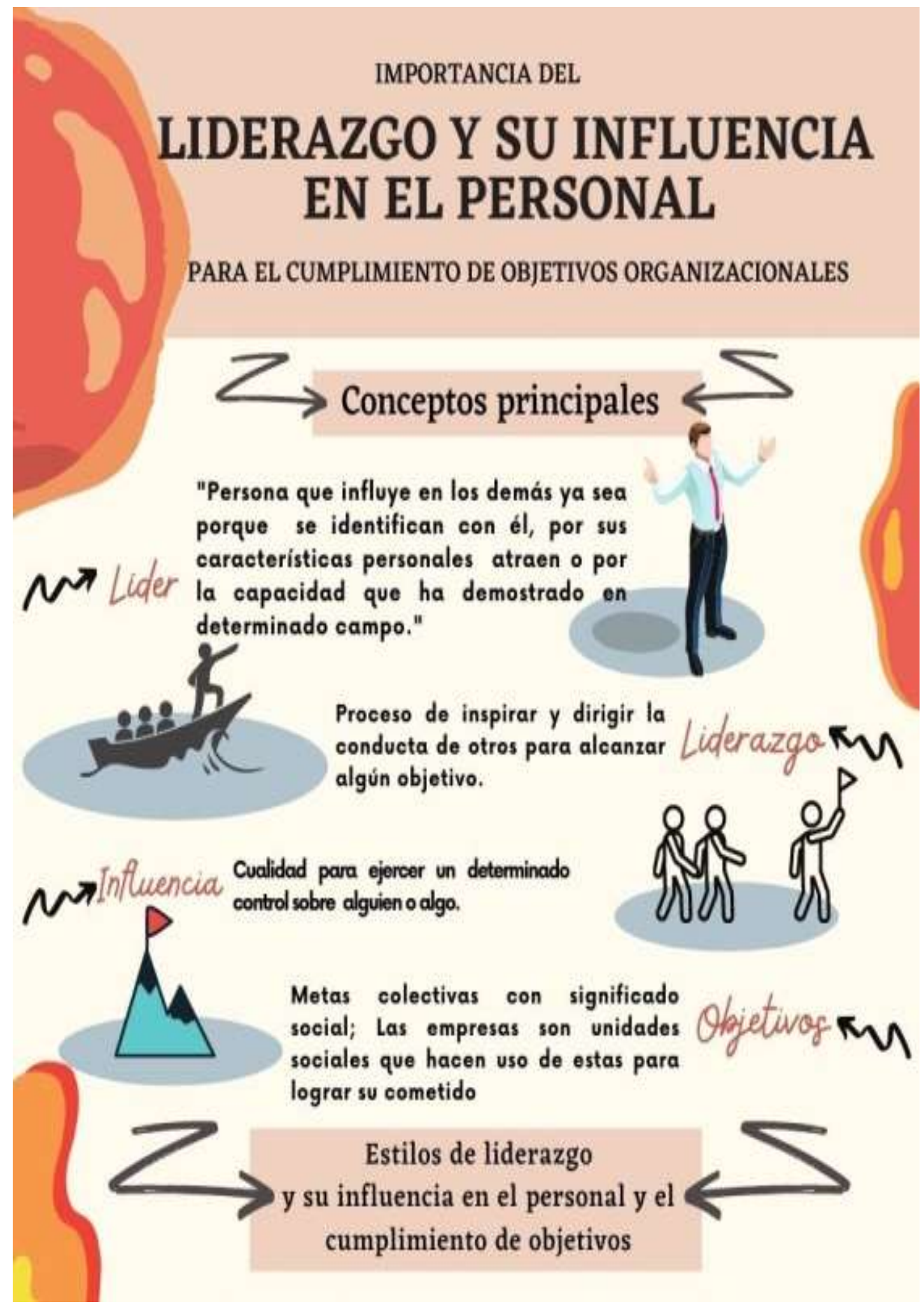


vit futocratica

Los subordinados pueden tomar decisiones, se involucran en las
metas, objetivos y procedimientos.

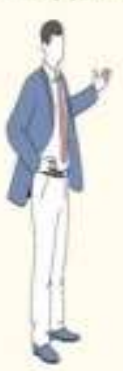

MRUEACIAPCPSONAL

-Sentimiento de engranaje en el cumplimiento de un objetivo sin importancia.

- Dependencia al lider para realizar una tarea.

\section{QzBETN 08}

Toma en cuenta a los integrantes, permite que generen nuevas ideas $y$ se involucren más actividades. participativo in

-Minimiza irresponsabilidad y los errores.

- Logro de ellos mediante técnicas y métodos.

-Mejora la eficiencia durante el proyecto.

\section{INRUEACIAPEPSORAL}

-La gestión participativa satisface la necesidad básica de seguridad, vinculación social, la estima $y$ el cumplimiento.

- Una mayor motivación, cohesión y el espiritu de equipo.

\section{QgzETN O\&}

- Aumento de productividad y eficiencia.

as - Los empleados son más voluntarios

$\int$ porque están más motivados.

$\int$ - Fusión de diferentes puntos de vista y $\int$ soluciones optimas.

$M$ Democrático

Los integrantes tienen libertad Autoridad centralizada que se dirige absoluta en la organización, de la cima a los niveles más bajos trabajan libremente y son parte de de la organización.

la toma de decisiones.

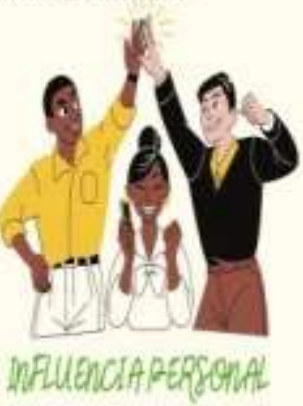

- Motivar a los empleados a ser tomados en cuenta.

-Existencia de reconocimiento por sus esfuernos.

-Sentimiento de ser útil ayudando y oolaborando.

\section{OPAETI Oo}

- Genera alto compromiso en los empleados. -Se delegan actividades $v$ responsabilidades.
Liberal

m

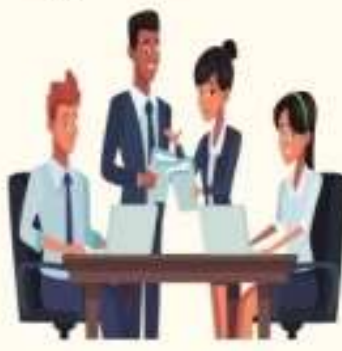

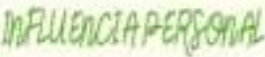

-Supone un incremento de la motivación entre los participantes. - Cada miembro tiene claras sus funciones y tiene libertad para completarlas.

\section{OPJET Nof}

Todos los integrantes del grupo son libres de manifestar sus opiniones y tomar decisiones según su interés y los de la compañía.

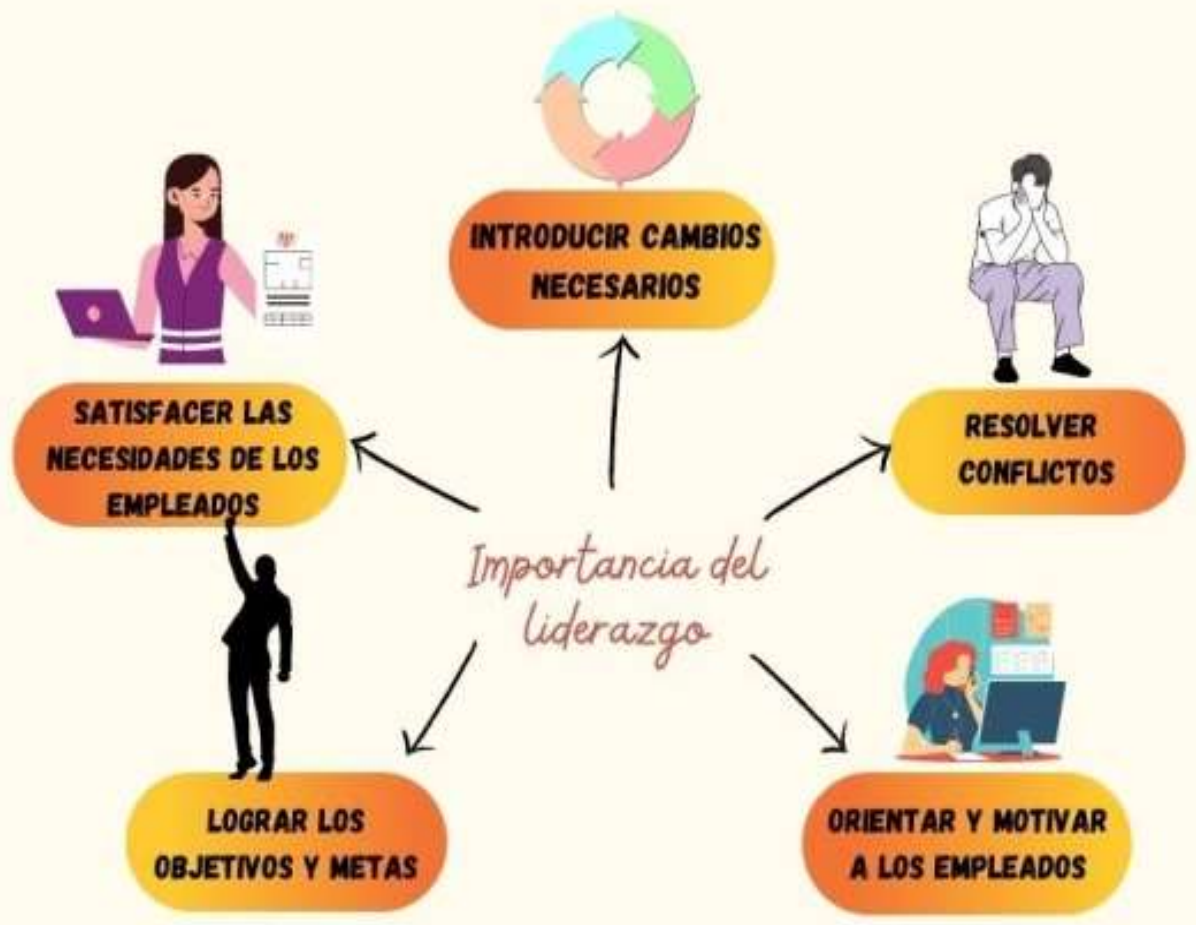




\section{Referencias}

[1] Chiavenato, I. (2009). Introducción a la teoría general de la administración (10th Edición). McGraw-Hill Interamericana. (Página 66- 76) https://bookshelf.vitalsource.com/books/9781456269838

[2] Chiavenato, I. (2017). Administración de recursos humanos (10th Edición). McGraw-Hill Interamericana. https://bookshelf.vitalsource.com/books/9781456263430

[3] Delpierre, J. F. (diciembre 2009). Modelo de liderazgo participativo para mejorar la calidad total. Instituto Tecnológico y de Estudios Superiores de Monterrey.

[4] Escandon-Barbosa, D. M., \& Hurtado-Ayala, A. (2016). Influencia de los estilos de liderazgo en el desempeño de las empresas exportadoras colombianas. Estudios gerenciales, 32(139), 137- 145.

[5] Fuentes, E. (2019, julio 10). Liderazgo Democrático Características, ventajas e inconvenientes.Liderexponencial.es.

https://liderexponencial.es/liderazgo-democratico-caracteristicas-

ventajas-e-inconvenientes/

[6] Liderazgo liberal. (2019, julio22). Economipedia.com https://economipedia.com/definiciones/liderazgo-liberal.html

[7] Madrigal, B. (2017). Habilidades directivas (3rd Edición). McGrawHill Interamericana.

https://bookshelf.vitalsource.com/books/9781456258535

[8] Pañeda, R.J. B. (2014). Administración (2nd Edición). McGraw-Hill Interamericana. https://bookshelf.vitalsource.com/books/9781456211196 